\title{
Robust Fault-tolerant Iterative Learning Control for Discrete Systems via Linear Repetitive Processes Theory
}

\author{
Jian Ding ${ }^{1} \quad$ Blazej Cichy ${ }^{2} \quad$ Krzysztof Galkowski $^{2} \quad$ Eric Rogers $^{3} \quad$ Hui-Zhong Yang ${ }^{1}$ \\ ${ }^{1}$ Key Laboratory of Advanced Process Control for Light Industry (Ministry of Education), Jiangnan University, Wuxi 214122, China \\ ${ }^{2}$ Institute of Control and Computation Engineering, University of Zielona Gora, Podgorna Street 50, 65-246 Zielona Gora, Poland \\ ${ }^{3}$ Department of Electronics and Computer Science, University of Southampton, Southampton SO17 1BJ, UK
}

\begin{abstract}
This paper addresses the problem of robust iterative learning control design for a class of uncertain multiple-input multipleoutput discrete linear systems with actuator faults. The stability theory for linear repetitive processes is used to develop formulas for gain matrices design, together with convergent conditions in terms of linear matrix inequalities. An extension to deal with model uncertainty of the polytopic or norm bounded form is also developed and an illustrative example is given.
\end{abstract}

Keywords: Iterative learning control, linear repetitive processes, linear matrix inequality (LMI), discrete linear systems, fault-tolerant control.

\section{Introduction}

Many industrial applications consist of repeated execution of the same task over a finite duration. A generic example is a gantry robot executing a pick and place task where the sequence of operations is: 1) collect the payload from a fixed location, 2) transfer the payload over a finite duration, 3) place the payload at a fixed location or under synchronization on a moving conveyor, 4) return to the starting location and 5) repeat 1)-4) as many times as required or until a halt is required for maintenance or for other reasons. Each execution is known as a trial over the finite trial length. Once a trial is complete, all the information generated during execution is available for use in computing the control input for the next trial.

Iterative learning control (ILC) was developed for applications such as the gantry robot task briefly outlined above where previous trial information is used to compute the control input to be applied on the next trial. The basic idea is to improve performance from trial-to-trial and non-causal temporal information can be used provided it is generated in a previous trial. For example, at instant, say $t$, in trial $k+1, k \geq 0$, information at $t+\lambda, \lambda>0$, on trial $k$ can be used in the computation of the control input on subsequent trials. The use of such information is the main distinguishing feature of ILC.

The widely recognized starting point for ILC research is [1], which considered a simple first order linear servomecha-

\footnotetext{
Regular paper

Special Issue on Latest Advances in ILC/RLC Theory and Applications

Manuscript received April 28, 2014; accepted October 28, 2014

This work was supported by National Natural Science Foundation of

China (Nos. 61273070 and 61203092) and 111 project (No. B12018).

Recommended by Guest Editor Rong-Hu Chi

(C) Institute of Automation, Chinese Academy of Science and Springer-Verlag Berlin Heidelberg 2015
}

nism system for speed control of a voltage-controlled direct current servomotor. Since this first work, ILC has been broadened in breadth and depth, for recent overviews of the literature $[2,3]$. Robust ILC design is an active topic lately ${ }^{[4-7]}$. Applications of ILC included batch-to-batch control $^{[8,9]}$, traffic control ${ }^{[10,11]}$, point-to-point control ${ }^{[12]}$, and positioning control ${ }^{[13-14]}$.

In this paper, we exploit the natural 2D system structure of ILC where one direction of information is from trial-totrial indexed by the subscript $k$ and the other along a trial indexed by $p$. The first work on treating ILC in a $2 \mathrm{D}$ system setting is [15], where the Roesser state-space model is used. This initial work has been followed by extensions to deal with, e.g., uncertainty in the model of the dynamics and uncertain initial conditions at the start of each trial ${ }^{[16]}$. In [17], the 2D system setting for analysis and design has been extended to nonlinear batch processes operating in a series of piecewise affine operating regions and with industrial applications in mind, allowing the presence of constraints on the input and output.

Given that the trial length is finite by definition, a more natural model for ILC is a repetitive process ${ }^{[18]}$. These processes are characterized by a series of sweeps, termed as passes, through a set of dynamics defined over a fixed finite duration known as the pass length. In particular, a pass is completed and then the process is reset before the start of the next pass. On each pass, an output, termed the pass profile, is produced which acts as a forcing function on, and hence contributes to, the dynamics of the next pass profile.

Repetitive processes have their origins in the coal mining industry, references to the original work in the 1970s are given in [18], where coal is extracted by a cutting machine that makes repeated sweeps along the coal face. During any sweep, the machine rests on the previous pass profile, the 
height of the stone/coal interface above some datum line. The basic geometry confirms that coal cutting is a repetitive process and other physical examples are again discussed in [18] and the relevant cited references.

The explicit interaction between successive pass profiles in a repetitive process can lead to oscillations that increase in amplitude from pass-to-pass and these oscillations cannot be removed by standard control action. A stability theory for linear constant pass length repetitive processes has been developed ${ }^{[18]}$ that, in essence, requires that a bounded initial pass profile produces a bounded sequence of pass profiles. This theory is based on an abstract model of the dynamics in a Banach space setting with the bounded property defined in terms of the norm of the Banach space to which the pass profile is assumed to belong. The repetitive process setting has been used to design ILC laws that have, in contrast to the $2 \mathrm{D}$ system based designs, been experimentally verified on a gantry robot ${ }^{[19,20]}$, where the necessary computations are linear matrix inequality (LMI) based.

In [21], the generalized Kalman-Yakubovich-Popov Lemma is used to develop ILC design algorithms in the repetitive process setting where frequency attenuation, the physical interpretation of the stability property, is only imposed over finite frequency ranges selected by knowledge of the particular application, again with experimental verification. Also, the repetitive process setting has been used ${ }^{[22]}$ to develop results on how to make the most effective use of previous trial information.

In operation, an ILC control law requires, as in other areas, that actuators and sensors function properly, where should a failure occur this can be total, in which case the overall control scheme may fail, or partial. One way to guard against such problems is to do a fault tolerant design ${ }^{[23]}$. This topic has already been the subject of productive research in ILC $^{[24-26]}$. This paper contributes to this general area by using the repetitive process setting to analyze the effects of actuator faults, leading to LMI based control law design algorithms with an extension to both norm bounded and polytopic based robustness. A numerical case study is also given.

The next section gives the required background on repetitive processes and formulates the general problem considered, including the representation of the faults. Section 3 then considers nominal model based ILC design and Section 4 extends the analysis to norm bounded and polytopic uncertainty. In Section 5, a simulation based case study is given using the model of an electric motor system used for evaluating other ILC designs. Section 6 concludes the paper by giving an overview of the new results obtained and discussion of possible future research.

Throughout this paper, the null and identity matrices of compatible dimensions are denoted by 0 and $I$ respectively. Also, $X>0$ and $X<0$ denote a symmetric positive definite matrix and a symmetric negative definite matrix, respectively. The symbol $*$ represents transposed entries in a symmetric matrix and $\rho(\cdot)$ denotes the spectral radius of its matrix argument.

\section{Background and problem formula- tion}

Repetitive processes are characterized by a series of sweeps, termed passes, through a set of dynamics defined over a finite duration known as the pass length. On each pass, an output produced termed the pass profile and the sequence of operations is that a pass is completed and then the process resets to the starting location and the next pass can begin, either immediately or after some time has elapsed. The pass profile produced on the previous pass acts as forcing function on the next pass and hence contributes to its dynamics. Variables in a repetitive process are two dimensional functions and the notation used is of the form $y_{k}(p), 0 \leq p \leq \alpha-1, k \geq 0$, where $y$ is the scalar or vector valued variable, $\alpha<\infty$ is the pass length and the subscript $k$ denotes the pass number.

Let $\left\{y_{k}\right\}$ denote the sequence of pass profiles generated by an example. Then, the control problem is that this sequence can contain oscillations that increase in amplitude from pass-to-pass, i.e., with $k$. Repetitive processes have their origins in the coal mining industry where coal is mined by a series of passes along the coal face by a cutting machine that rests on the previous pass profile during the completion of the next pass. The original references are in [18], and in this example, the oscillations in the pass profile sequence are due, in the main, to the weight of the cutting machine. In the absence of control action, the only option is to halt productive work to enable their manual removal.

Pass-to-pass oscillations generated by a repetitive process cannot be controlled by application of standard, or 1D, control system theory and design since such an approach ignores their $2 \mathrm{D}$ system structure, i.e., the finite pass length and the resetting of the initial conditions before the start of each new pass. A complete treatment of this aspect can be found in [18] and the relevant cited references.

A stability theory for linear constant pass length repetitive processes has been developed ${ }^{[18]}$. This is based on an abstract model in a Banach space setting that includes wide range of such linear repetitive processes. Given the control problem, this theory demands that a bounded initial pass profile produces as bounded sequence of pass profiles, with boundedness defined in terms of the norm on the underlying function space. Moreover, this property can be enforced over the finite and fixed pass length, termed asymptotic stability, or for all possible values of the finite pass length, termed stability along the pass. This last property can be analyzed by considering $\alpha \rightarrow \infty$ and this is the form of repetitive process stability required in this paper.

The ILC design in this paper is based on discrete linear repetitive processes whose state-space model over $0 \leq p \leq$ 


$$
\begin{aligned}
\alpha-1, & k \geq 0, \text { is } \\
& x_{k+1}(p+1)=\boldsymbol{A} x_{k+1}(p)+\boldsymbol{B} u_{k+1}(p)+\boldsymbol{B}_{0} y_{k}(p) \\
& y_{k+1}(p)=\boldsymbol{C} x_{k+1}(p)+\boldsymbol{D} u_{k+1}(p)+\boldsymbol{D}_{0} y_{k}(p)
\end{aligned}
$$

where on pass $k, x_{k}(p) \in \mathbf{R}^{n}$ is the state vector, $y_{k}(p) \in \mathbf{R}^{m}$ is the pass profile vector and $u_{k}(p) \in \mathbf{R}^{r}$ is the control input vector. The boundary conditions, i.e., the pass state initial vector sequence and the initial pass profile, are

$$
\begin{array}{ll}
x_{k+1}(0)=d_{k+1}, & k \geq 0, \\
y_{0}(p)=f(p), & 0 \leq p \leq \alpha-1
\end{array}
$$

where the vector functions $d_{k+1}$ and $f(p)$ have known entries. In this model, it is not assumed that the entries in $d_{k+1}$ are the same for each $k$ but they are independent of the previous pass dynamics. These boundary conditions are the simplest possible but applications do exist where they must be replaced by an alternative structure and, in particular, the entries of a state initial vector could be set as functions of sampling instants on each pass. This topic is also treated in [18] where the abstract model based theory shows that such state initial conditions alone can cause instability.

As discussed above, stability of these processes is defined in terms of the contribution of the previous pass profile to that on the next pass. For processes described by (1) and (2), the pass-to-pass coupling is described by the convolution operator, denoted by $L_{\alpha}$, for a standard linear system with state-space model matrices $\left\{\boldsymbol{A}, \boldsymbol{B}_{0}, \boldsymbol{C}, \boldsymbol{D}_{0}\right\}$. Hence the contribution from pass $k$ to pass $k+1$ can be written as $y_{k+1}=L_{\alpha} y_{k}, k \geq 0$. Also, let $y_{k} \in E_{\alpha}$, where $E_{\alpha}$ is a suitably chosen Banach space with norm denoted by $\|\cdot\|$ and let the same symbol denote the induced norm on the bounded linear operator $L_{\alpha}$.

Asymptotic stability for linear repetitive processes is equivalent to the existence of finite real scalars $M_{\alpha}>0$ and $\lambda_{\alpha} \in(0,1)$ such that $\left\|L_{\alpha}^{k}\right\| \leq M_{\alpha} \lambda_{\alpha}^{k}, k \geq 0$. For examples, described by (1) and (2), asymptotic stability requires that $\rho\left(\boldsymbol{D}_{0}\right)<1$ and if this condition holds, the sequence of pass profiles produced will converge in $k$ to a standard discrete linear system, termed the limit profile, with state matrix $\boldsymbol{A}_{l p}=\boldsymbol{A}+\boldsymbol{B}_{0}\left(I-\boldsymbol{D}_{0}\right)^{-1} \boldsymbol{C}$. However, consider the special case when $\boldsymbol{A}=0.5, \boldsymbol{B}=1, \boldsymbol{B}_{0}=-0.5+\beta, \boldsymbol{C}=1$ and $\boldsymbol{D}_{0}=0$. Then $\boldsymbol{A}_{l p}=\beta$ and hence the limit profile is unstable in $p$, i.e., along the pass when $|\beta| \geq 1$.

The existence of examples such as the one given above is due to the finite pass length, a property of the process and not an assumption imposed for analysis purposes. Stability along the pass prevents such examples from arising by imposing the stability property for all possible values of the finite pass length. This property requires the existence of finite real scalars existence of finite real scalars $M_{\infty}>0$ and $\lambda_{\infty} \in(0,1)$, which are independent of $\alpha$, such that $\left\|L_{\alpha}^{k}\right\| \leq M_{\infty} \lambda_{\infty}^{k}, k \geq 0$. The following result characterizes stability along the pass of examples described by (1) and (2).

Lemma $\mathbf{1}^{[18]}$. A discrete linear repetitive process de- scribed by (1) and (2) is stable along the pass if and only if 1) $\left.\rho\left(\boldsymbol{D}_{0}\right)<1,2\right) \rho(\boldsymbol{A})<1$, and 3) all eigenvalues of $G(z)=\boldsymbol{C}(z I-\boldsymbol{A})^{-1} \boldsymbol{B}_{0}+\boldsymbol{D}_{0}$ have modulus strictly less than unity for all $|z|=1$.

Note that the intuitively obvious condition 2) in this result is also only a necessary condition for stability along the pass as the simple example given above demonstrates. Condition 3) in this result requires frequency attenuation of the complete spectrum of the previous pass profile.

The systems considered in this paper have the following state-space model in the ILC setting

$$
\begin{aligned}
& x_{k}(p+1)=A x_{k}(p)+B u_{k}(p) \\
& y_{k}(p)=C x_{k}(p) \\
& x_{k+1}(0)=d_{k+1}, \quad 0 \leq p \leq \alpha-1
\end{aligned}
$$

where on trial $k, x_{k}(p) \in \mathbf{R}^{n}$ is the state vector, $y_{k}(p) \in \mathbf{R}^{m}$ is the output vector, $u_{k}(p) \in \mathbf{R}^{r}$ and the entries in $d_{k+1}$ are known constants. Also let $u_{k, i}(p), 1 \leq i \leq r$, denote the entries in $u_{k}(p)$ and let $u_{k, i}^{F}(p)$ denote an input and actuator signal with possible failures. Then, the failure model used in this paper is ${ }^{[24]}$

$$
u_{k, i}^{F}(p)=\gamma_{i} u_{k, i}(p), i=1,2, \cdots, r
$$

where

$$
0 \leq \underline{\gamma}_{i} \leq \gamma_{i} \leq \bar{\gamma}_{i}, i=1,2, \cdots, r
$$

The scalars $\underline{\gamma}_{i}\left(\underline{\gamma}_{i} \leq 1\right)$ and $\bar{\gamma}_{i}\left(\bar{\gamma}_{i} \geq 1\right)$ in this failure model are assumed to be known. The scalar $\gamma_{i}$ is unknown but assumed to vary within a known range. If $\underline{\gamma}_{i}=\bar{\gamma}_{i}$, the fault-free case is recovered, i.e., $u_{i}^{F}=u_{i}$. Also, $\gamma_{i}=0$ is the case when the actuator experiences complete failure and $\gamma_{i}>0$ corresponds to a partial failure, i.e., partial degradation of the actuator.

Introduce the notation

$$
\begin{aligned}
& \Gamma=\operatorname{diag}\left\{\gamma_{1}, \gamma_{2}, \cdots, \gamma_{r}\right\} \\
& \bar{\Gamma}=\operatorname{diag}\left\{\bar{\gamma}_{1}, \bar{\gamma}_{2}, \cdots, \bar{\gamma}_{r}\right\} \\
& \underline{\Gamma}=\operatorname{diag}\left\{\underline{\gamma}_{1}, \underline{\gamma}_{2}, \cdots, \underline{\gamma}_{r}\right\}
\end{aligned}
$$

to give

$$
u_{k}^{F}=\left[u_{k, 1}^{F}, u_{k, 2}^{F}, \cdots, u_{k, r}^{F}\right]^{\mathrm{T}}=\Gamma u_{k} .
$$

Hence, the system described by (3) with actuator faults of the form considered in this paper can be described by the state-space model

$$
\begin{aligned}
& x_{k}(p+1)=A x_{k}(p)+B \Gamma u_{k}(p) \\
& y_{k}(p)=C x_{k}(p) \\
& x_{k+1}(0)=d_{k+1}, \quad 0 \leq p \leq \alpha-1 .
\end{aligned}
$$

Also, let $y_{r}(p)$ denote the supplied reference vector. Then, the tracking error on trial $k$ is

$$
e_{k}(p)=y_{r}(p)-y_{k}(p) \text {. }
$$

The control design problem is to determine a faulttolerant ILC law such that trial-to-trial error convergence 
occurs in $k$, i.e., $\lim _{k \rightarrow \infty}\left\|e_{k}\right\|=0$, where $\|\cdot\|$ is a convenient norm, in the presence of actuator failures. Moreover, the control input applied on each trial must satisfy $\lim _{k \rightarrow \infty}\left\|u_{k}-u_{\infty}\right\|=0$, where $u_{\infty}$ is termed the learned control.

The following structure for the computation of the current trial input permits the use of previous trial data

$$
u_{k+1}(p)=u_{k}(p)+\Delta u_{k+1}(p)
$$

i.e., the control input on the previous trial plus a correction term $\Delta u_{k+1}(p)$. Introduce, for analysis purposes only,

$$
\eta_{k+1}(p+1)=x_{k+1}(p)-x_{k}(p)
$$

and set

$$
\Delta u_{k+1}(p)=K_{1} \eta_{k+1}(p+1)+K_{2} e_{k}(p+1)
$$

where $K_{1}$ and $K_{2}$ are compatibly dimensioned matrices to be designed. Then, the controlled dynamics can be reformulated as

$$
\begin{gathered}
\eta_{k+1}(p+1)=A x_{k+1}(p-1)+B \Gamma u_{k+1}(p-1)- \\
A x_{k}(p-1)-B \Gamma u_{k}(p-1)= \\
\left(A+B \Gamma K_{1}\right) \eta_{k+1}(p)+B \Gamma K_{2} e_{k}(p) \\
e_{k+1}(p)=y_{d}(p)-y_{k+1}(p)= \\
e_{k}(p)+y_{k}(p)-y_{k+1}(p)= \\
-C\left(A+B \Gamma K_{1}\right) \eta_{k+1}(p)+ \\
\left(I-C B \Gamma K_{2}\right) e_{k}(p)
\end{gathered}
$$

or

$$
\begin{aligned}
& \eta_{k+1}(p+1)=\widehat{A} \eta_{k+1}(p)+\widehat{B} e_{k}(p) \\
& e_{k+1}(p)=\widehat{C} \eta_{k+1}(p)+\widehat{D} e_{k}(p)
\end{aligned}
$$

where

$$
\begin{aligned}
& \widehat{A}=A+B \Gamma K_{1}, \widehat{B}=B \Gamma K_{2}, \\
& \widehat{C}=-C\left(A+B \Gamma K_{1}\right), \widehat{D}=I-C B \Gamma K_{2}
\end{aligned}
$$

The state-space model (13) is that of a discrete linear repetitive process of the form (1) with the input term deleted from both the state and pass profile updating equations. On trial $k+1$, the ILC error $e_{k+1}(p)$ is the pass profile vector and $\eta_{k+1}(p)$ is the current state vector.

In the next section, repetitive process theory is applied to ILC design, where for the remainder of this paper, pass is used instead of trial to conform with repetitive process literature.

\section{ILC design}

Introduce the notation

$$
\begin{aligned}
& \Sigma=\operatorname{diag}\left\{\sigma_{1}, \sigma_{2}, \cdots, \sigma_{r}\right\} \\
& \Sigma_{0}=\operatorname{diag}\left\{\sigma_{10}, \sigma_{20}, \cdots, \sigma_{r 0}\right\}
\end{aligned}
$$

with

$$
\begin{gathered}
\sigma_{i}=\frac{\bar{\gamma}_{i}+\underline{\gamma}_{i}}{2}, i=1,2, \cdots, r \\
\sigma_{i 0}=\frac{\bar{\gamma}_{i}-\underline{\gamma}_{i}}{\bar{\gamma}_{i}+\underline{\gamma}_{i}}, i=1,2, \cdots, r .
\end{gathered}
$$

Also, from (6) and (15), an unknown matrix $\Gamma_{0}$ exists such that

$$
\Gamma=\left(I+\Gamma_{0}\right) \Sigma
$$

and

$$
\left|\Gamma_{0}\right| \leq \Sigma_{0} \leq I
$$

where

$$
\Gamma_{0}=\operatorname{diag}\left\{\gamma_{10}, \gamma_{20}, \cdots, \gamma_{r 0}\right\}
$$

and

$$
\left|\Gamma_{0}\right|=\operatorname{diag}\left\{\gamma_{10}|,| \gamma_{20}|, \cdots,| \gamma_{r 0}\right\}
$$

The analysis that follows will make extensive use of the Schur's complement formula and the following result.

Lemma $2^{[27]}$. Assume $X, Y, Z=Z^{\mathrm{T}}$ are real matrices with compatible dimensions. Then, for any matrix $\Delta$ satisfying $\Delta^{\mathrm{T}} \Delta \leq I$, the inequality

$$
Z+X \Delta Y+Y^{\mathrm{T}} \Delta^{\mathrm{T}} X^{\mathrm{T}}<0
$$

holds if and only if there exists a positive scalar $\varepsilon$ such that

$$
Z+\varepsilon X X^{\mathrm{T}}+\varepsilon^{-1} Y^{\mathrm{T}} Y<0 .
$$

The conditions of Lemma 1 can be used to test the stability of the controlled dynamics (13) and also have well defined physical interpretations. In particular, condition 1) of this result shows that ILC pass-to-pass error convergence is independent of the state initial vector sequence assumed in this work. However, if the initial state vector is a function of points along the previous trial, e.g., $x_{k+1}(0)=d_{k+1}+L y_{k}(\alpha-1)$, then repetitive process stability theory ${ }^{[18]}$, shows this term alone can cause instability and hence pass-to-pass error divergence would occur. Condition 2) of Lemma 1 guarantees that state dynamics along any pass are bounded independent of the pass length and condition 3) imposes frequency attenuation over the complete spectrum of the previous pass error.

An alternative to Lemma 1 is to use a Lyapunov functionbased interpretation of stability ${ }^{[18]}$, which results in LMI based design where the computations to be completed involve only matrices with constant entries. This approach is based on a sufficient, but not necessary, set of stability conditions, but designs in this setting have been experimentally verified ${ }^{[19,20]}$. The following is the first new result of this paper.

Theorem 1. The discrete linear repetitive process representation of the ILC dynamics (13) is stable along the 
pass if there exists a scalar $\lambda>0$ and matrices $X_{1}>0$, $X_{2}>0, R_{1}$ and $R_{2}$ such that the following LMI is feasible:

$$
\left[\begin{array}{ccc}
-X_{1} & * & \\
0 & -X_{2} & \\
A X_{1}+B \Sigma R_{1} & B \Sigma R_{2} & \\
-C A X_{1}-C B \Sigma R_{1} & X_{2}-C B \Sigma R_{2} & \\
\Sigma R_{1} & \Sigma R_{2} & \\
* & * & * \\
* & * & * \\
-X_{1}+\lambda B \Sigma_{0}^{2} B^{\mathrm{T}} & * & * \\
-\lambda C B \Sigma_{0}^{2} B^{\mathrm{T}} & -X_{2}+\lambda C B \Sigma_{0}^{2} B^{\mathrm{T}} C^{\mathrm{T}} & * \\
0 & 0 & -\lambda I
\end{array}\right]<0 .
$$

If this LMI is feasible, the control law matrices $K_{1}$ and $K_{2}$ can be computed using

$$
\begin{aligned}
& K_{1}=R_{1} X_{1}^{-1} \\
& K_{2}=R_{2} X_{2}^{-1} .
\end{aligned}
$$

Proof. Consider the candidate Lyapunov function

$$
V_{k}(p)=V_{k}^{1}(p)+V_{k}^{2}(p)
$$

where

$$
\begin{aligned}
V_{k}^{1}(p) & =\eta_{k+1}^{\mathrm{T}}(p) P_{1} \eta_{k+1}(p) \\
V_{k}^{2}(p) & =e_{k}^{\mathrm{T}}(p) P_{2} e_{k}(p) .
\end{aligned}
$$

with $P_{i}>0, i=1,2$ and increment

$$
\begin{aligned}
\Delta V_{k}(p)= & \Delta V_{k}^{1}(p)+\Delta V_{k}^{2}(p)= \\
& V_{k}^{1}(p+1)-V_{k}^{1}(p)+V_{k+1}^{2}(p)-V_{k}^{2}(p)= \\
& {\left[\begin{array}{cc}
\eta_{k+1}^{\mathrm{T}}(p) & e_{k}^{\mathrm{T}}(p)
\end{array}\right] \times } \\
& \left(\Phi^{\mathrm{T}} P \Phi-P\right)\left[\begin{array}{c}
\eta_{k+1}(p) \\
e_{k}(p)
\end{array}\right]
\end{aligned}
$$

where $\Phi=\left[\begin{array}{cc}\widehat{A} & \widehat{B} \\ \widehat{C} & \widehat{D}\end{array}\right], P=\operatorname{diag}\left\{P_{1}, P_{2}\right\}$. Then, (13) is stable along the pass if there exists $P>0$ such that

$$
\Phi^{\mathrm{T}} P \Phi-P<0 .
$$

An obvious application of the Schur's complement formula to (23) yields

$$
\left[\begin{array}{cccc}
-P_{1} & * & * & * \\
0 & -P_{2} & * & * \\
\widehat{A} & \widehat{B} & -P_{1}^{-1} & * \\
\widehat{C} & \widehat{D} & 0 & -P_{2}^{-1}
\end{array}\right]<0
$$

Next, introduce

$$
X_{1}=P_{1}^{-1}, \quad X_{2}=P_{2}^{-1}
$$

and pre-(left) and post-(right) multiply (24) by $\operatorname{diag}\left\{X_{1}, X_{2}, I, I\right\}$ to obtain

$\left[\begin{array}{cccc}-X_{1} & * & * & * \\ 0 & -X_{2} & * & * \\ A X_{1}+B \Gamma K_{1} X_{1} & B \Gamma K_{2} X_{2} & -X_{1} & * \\ -C A X_{1}-C B \Gamma K_{1} X_{1} & X_{2}-C B \Gamma K_{2} X_{2} & 0 & -X_{2}\end{array}\right]<0$.

Also, from (16),

$$
\Lambda+H \Gamma_{0} F+F^{\mathrm{T}} \Gamma_{0}^{\mathrm{T}} H^{\mathrm{T}}<0
$$

where

$\left[\begin{array}{cccc}-X_{1} & * & * & * \\ 0 & -X_{2} & * & * \\ A X_{1}+B \Sigma K_{1} X_{1} & B \Sigma K_{2} X_{2} & -X_{1} & * \\ -C A X_{1}-C B \Sigma K_{1} X_{1} & X_{2}-C B \Sigma K_{2} X_{2} & 0 & -X_{2}\end{array}\right]$

$H=\left[\begin{array}{llll}0 & 0 & B^{\mathrm{T}} & -B^{\mathrm{T}} C^{\mathrm{T}}\end{array}\right]^{\mathrm{T}}$

$F=\left[\begin{array}{llll}\Sigma K_{1} X_{1} & \Sigma K_{2} X_{2} & 0 & 0\end{array}\right]$.

From Lemma 2, it follows that (26) holds if there exists a positive scalar $\lambda>0$ such that

$$
\Lambda+\lambda H \Sigma_{0}^{2} H^{\mathrm{T}}+\lambda^{-1} F^{\mathrm{T}} F<0 .
$$

Using the Schur's complement formula, (27) is equivalent to

$$
\left[\begin{array}{cc}
\Lambda+\lambda H \Sigma_{0}^{2} H^{\mathrm{T}} & * \\
F & -\lambda I
\end{array}\right]<0 .
$$

Finally, introducing

$$
R_{1}=K_{1} X_{1}, \quad R_{2}=K_{2} X_{2}
$$

completes the proof.

\section{Robustness}

\subsection{Norm bounded uncertainty}

Consider the following discrete-time linear system in the ILC setting with actuator faults and possibly time-varying uncertainties

$$
\begin{aligned}
& x_{k}(p+1)=(A+\Delta A) x_{k}(p)+B \Gamma u_{k}(p) \\
& y_{k}(p)=C x_{k}(p) \\
& x_{k+1}(0)=d_{k+1}, \quad 0 \leq p \leq \alpha-1
\end{aligned}
$$

where $\Delta A$ represents admissible uncertainties that are assumed to satisfy

$$
\Delta A=H_{1} \Delta F_{1}
$$

where $H_{1}$ and $F_{1}$ are known constant matrices of appropriate dimensions and $\Delta$ is an unknown constant matrix or a matrix function satisfying

$$
\Delta^{\mathrm{T}} \Delta \leq I .
$$


Using (9)-(11) and following the analysis of the previous section, the controlled ILC dynamics with faults are described by the uncertain discrete linear repetitive process state-space model

$$
\begin{aligned}
& \eta_{k+1}(p+1)=(\widehat{A}+\Delta \widehat{A}) \eta_{k+1}(p)+\widehat{B} e_{k}(p) \\
& e_{k+1}(p)=(\widehat{C}+\Delta \widehat{C}) \eta_{k+1}(p)+\widehat{D} e_{k}(p)
\end{aligned}
$$

where $\Delta \widehat{A}=\Delta A$ and $\Delta \widehat{C}=-C \Delta A$.

Theorem 2. The discrete linear repetitive process representation of the ILC dynamics (32) is stable along the pass if there exist scalars $\lambda>0, \epsilon>0$ and matrices $X_{1}>0$, $X_{2}>0, R_{1}, R_{2}$ such that the following LMI is feasible:

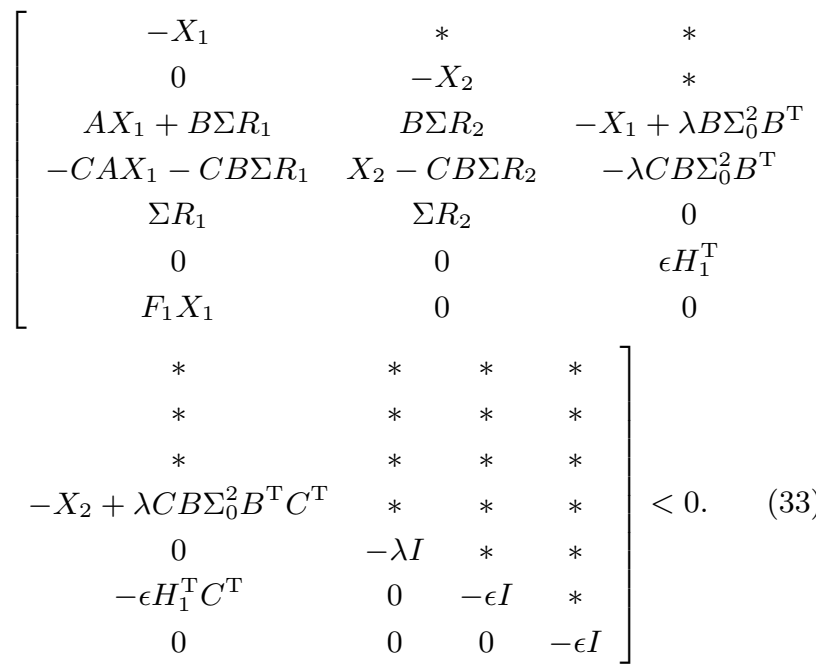

If this LMI is feasible, the control law matrices $K_{1}$ and $K_{2}$ are given by $K_{1}=R_{1} X_{1}^{-1}$ and $K_{2}=R_{2} X_{2}^{-1}$.

Proof. In addition to the proof for Theorem 1, it is required to show that

$$
\Pi+\boldsymbol{H} \Delta \boldsymbol{F}+\boldsymbol{F}^{\mathrm{T}} \Delta^{\mathrm{T}} \boldsymbol{H}^{\mathrm{T}}<0
$$

where

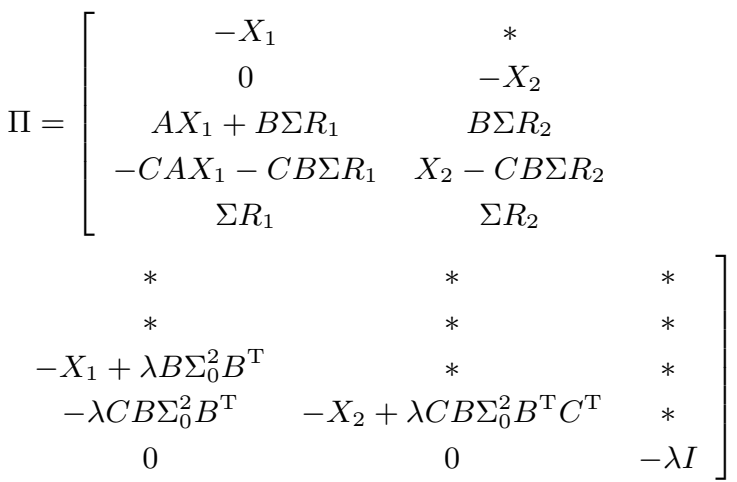

$$
\begin{aligned}
& \boldsymbol{H}=\left[\begin{array}{lllll}
0 & 0 & H_{1}^{\mathrm{T}} & -H_{1}^{\mathrm{T}} C^{\mathrm{T}} & 0
\end{array}\right]^{\mathrm{T}} \\
& \boldsymbol{F}=\left[\begin{array}{lllll}
F_{1} X_{1} & 0 & 0 & 0 & 0
\end{array}\right] \text {. }
\end{aligned}
$$

From Lemma 2, it follows that (34) holds for all $\Delta$ satisfying (31) if there exists a positive scalar $\epsilon>0$ such that

$$
\Pi+\epsilon \boldsymbol{H} \boldsymbol{H}^{\mathrm{T}}+\epsilon^{-1} \boldsymbol{F}^{\mathrm{T}} \boldsymbol{F}<0
$$

which, on applying the Schur's complement formula, is equivalent to

$$
\left[\begin{array}{cc}
\Pi & * \\
{\left[\begin{array}{c}
\epsilon^{\frac{1}{2}} \boldsymbol{H}^{\mathrm{T}} \\
\epsilon^{-\frac{1}{2}} \boldsymbol{F}
\end{array}\right]} & -I
\end{array}\right]<0 .
$$

Finally pre-multiplying and post-multiplying (36) by $\operatorname{diag}\left\{I, I, I, I, I, \epsilon^{\frac{1}{2}} I, \epsilon^{\frac{1}{2}} I\right\}$ gives the LMI of (33).

\subsection{Polytopic uncertainty}

Polytopic uncertainty assumes that the matrices defining the system state-space model lie in a convex bounded uncertain domain $\Omega$ of the form

$$
\begin{aligned}
\Omega= & \{[A(\xi(k, p)), B(\xi(k, p))] \mid[A(\xi(k, p)), B(\xi(k, p))]= \\
& \left.\sum_{i=1}^{N} \xi_{i}(k, p)\left(A_{i}, B_{i}\right), \xi_{i} \geq 0, \sum_{i=1}^{N} \xi_{i}(k, p)=1\right\}
\end{aligned}
$$

where $A_{i}, B_{i}$ are the corresponding matrix vertices and $N$ denotes their number.

The uncertain discrete linear repetitive process model for ILC in the presence of polytopic uncertainty is

$$
\begin{aligned}
& \eta_{k+1}(p+1)=\widehat{A}(\xi(k, p)) \eta_{k+1}(p)+\widehat{B}(\xi(k, p)) e_{k}(p) \\
& e_{k+1}(p)=\widehat{C}(\xi(k, p)) \eta_{k+1}(p)+\widehat{D}(\xi(k, p)) e_{k}(p)
\end{aligned}
$$

where

$$
\begin{aligned}
& \widehat{A}(\xi(k, p))=A(\xi(k, p))+B(\xi(k, p)) \Gamma K_{1} \\
& \widehat{B}(\xi(k, p))=B(\xi(k, p)) \Gamma K_{2} \\
& \widehat{C}(\xi(k, p))=-C\left[A(\xi(k, p))+B(\xi(k, p)) \Gamma K_{1}\right] \\
& \widehat{D}(\xi(k, p))=I-C B(\xi(k, p)) \Gamma K_{2} .
\end{aligned}
$$

Theorem 3. The repetitive process representing the ILC dynamics (38) is stable along the pass if there exists a scalar $\lambda>0$ and matrices $X_{1}>0, X_{2}>0, R_{1}, R_{2}$ such that the following LMI is feasible:

$$
\left[\begin{array}{ccc}
-X_{1} & * & \\
0 & -X_{2} & \\
A_{i} X_{1}+B_{i} \Sigma R_{1} & B_{i} \Sigma R_{2} & \\
-C A_{i} X_{1}-C B_{i} \Sigma R_{1} & X_{2}-C B_{i} \Sigma R_{2} & \\
\Sigma R_{1} & \Sigma R_{2} & \\
* & * & * \\
* & * & * \\
-X_{1}+\lambda B_{i} \Sigma_{0}^{2} B_{i}^{\mathrm{T}} & * & * \\
-\lambda C B_{i} \Sigma_{0}^{2} B_{i}^{\mathrm{T}} & -X_{2}+\lambda C B_{i} \Sigma_{0}^{2} B_{i}^{\mathrm{T}} C^{\mathrm{T}} & * \\
0 & 0 & -\lambda I
\end{array}\right]<0
$$

for $i=1,2, \cdots, N$.

If this LMI is feasible, the control law matrices $K_{1}$ and $K_{2}$ are given by $K_{1}=R_{1} X_{1}^{-1}$ and $K_{2}=R_{2} X_{2}^{-1}$. 
Proof. The uncertain process is stable along the pass if this property holds for every vertex. The proof now follows identical steps to that of Theorem 1 for each matrix vertex.

\section{Case study}

Consider the following model of an electric motor system given in [22] and references therein

$$
\begin{aligned}
& \dot{x}(t)=A_{c} x(t)+B_{c} u(t) \\
& y(t)=C_{c} x(t)
\end{aligned}
$$

where

$$
\begin{aligned}
A_{c} & =\left[\begin{array}{ccc}
-\frac{V_{B}}{J} & -\frac{3 n_{p} \phi_{m g}}{2 J} & 0 \\
0 & -\frac{1}{\gamma} & 0 \\
\frac{\omega_{b}}{2 \pi} & 0.81 & 0
\end{array}\right] \\
B_{c} & =\left[\begin{array}{c}
0 \\
\frac{1}{\gamma} \\
0
\end{array}\right] \\
C_{c} & =\left[\begin{array}{lll}
0 & 0 & 1
\end{array}\right]
\end{aligned}
$$

with the following nominal motor parameters

$$
\begin{aligned}
& V_{B}=0.0625, J=0.0267, n_{p}=2 \\
& \gamma=6.8 \times 10^{-4}, \phi_{m g}=0.9102, \omega_{b}=315.315 .
\end{aligned}
$$

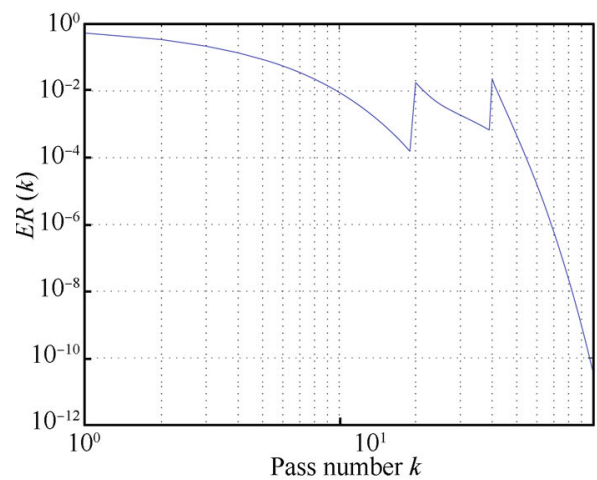

(a) Root mean square error

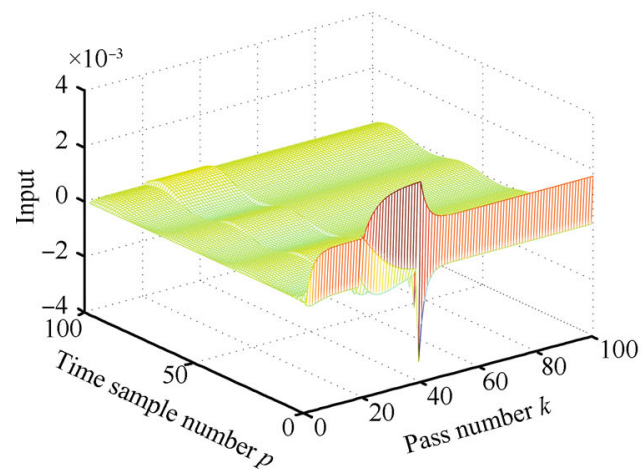

(c) Input progression
Also, this model has been sampled at $T_{s}=0.02 \mathrm{~s}$ to give a state-space model of the form (3). The pass length is $2 \mathrm{~s}$, the reference signal is

$$
y_{r}(t)=\sin (\pi t)+0.5 \sin (2 \pi t)+0.5 \sin (3 \pi t)
$$

and zero boundary conditions are assumed. Also, pass-topass error convergence is measured by the root mean square formula

$$
E R(k)=\sqrt{\frac{1}{\alpha} \sum_{p=0}^{\alpha-1} e_{k}^{\mathrm{T}}(p) e_{k}(p)} .
$$

Assume that there exists an unknown actuator fault $\Gamma$ but it is known that $0.7=\underline{\Gamma} \leq \Gamma \leq \bar{\Gamma}=1.3$, and hence from (14) and (15), $\Sigma=1, \Sigma_{0}=0.3$. The design is now illustrated by considering different scenarios of faults and uncertainty.

\subsection{Scenario 1: Constant fault and nomi- nal model}

The controlled nominal process has been simulated with $\Gamma=1.3$ until $k=20$ when a constant fault caused a switch to $\Gamma=0.7$, and then at $k=40$, a further switch returned $\Gamma$ to its starting value.

Application of Theorem 1 results in the control law matrices given in the first row of Table 1. The simulation results for the controlled dynamics are shown in Fig. 1.

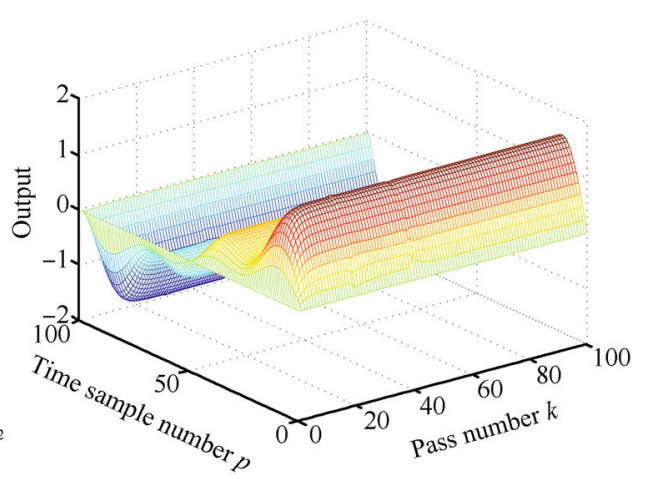

(b) Output progression

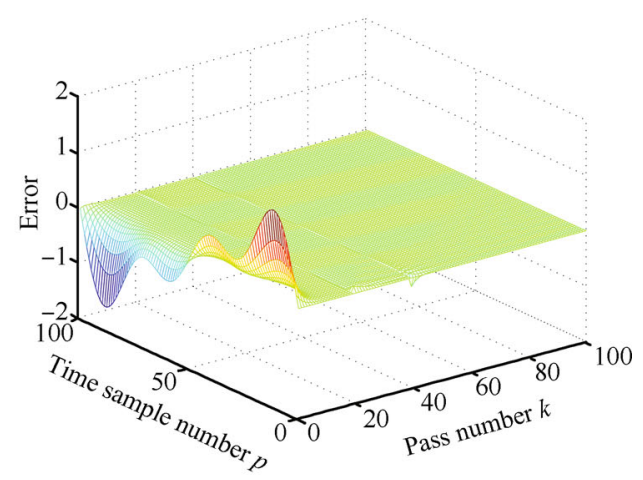

(d) Error progression 


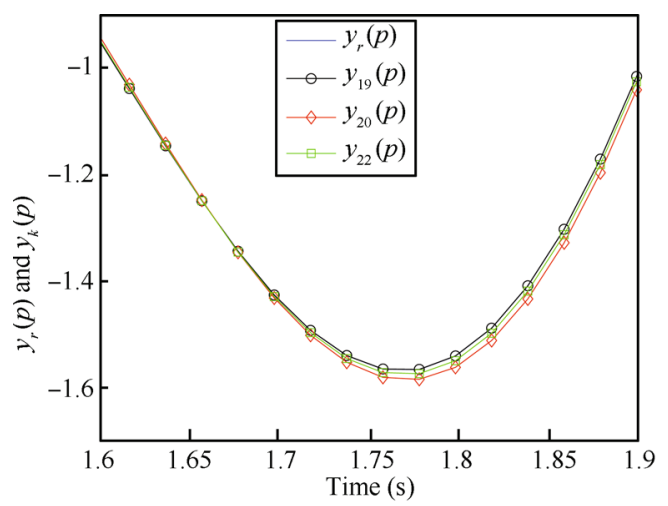

(e) A segment of $y_{r}(p)$ and $y_{k}(p)(k=19,20,22)$

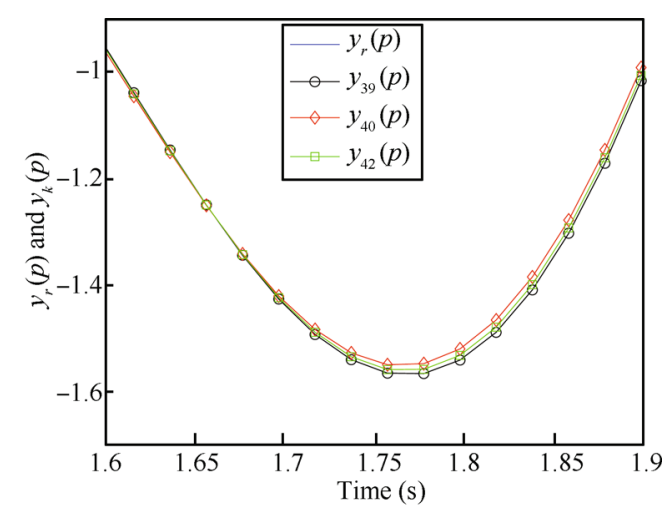

(f) A segment of $y_{r}(p)$ and $y_{k}(p)(k=39,40,42)$

Fig. 1 Simulation results for the Scenario 1 design

Table 1 Control law matrices

\begin{tabular}{ccccc}
\hline & $K_{1}$ & $K_{2}$ \\
\hline Scenario 1 & {$[-0.0099$} & -0.0007 & $-0.0079]$ & 0.0029 \\
Scenario 2 & {$[-0.0098$} & -0.0007 & $-0.0068]$ & 0.0014 \\
Scenario 3 & {$[-0.0080$} & -0.0007 & $-0.0062]$ & 0.0023 \\
\hline
\end{tabular}

\subsection{Scenario 2: Time-varying fault and model with norm-bounded uncertain- ties}

The controlled processes with norm-bounded uncertainty has been simulated with $\Gamma=0.7$ until $k=20$ when the constant fault switched to the time-varying fault $\Gamma=$ $1+0.3 \sin (p)$, and then at $k=40$, a further switch to $\Gamma=1+0.3 \cos (p)$ occurred. The matrices defining the uncertainty were taken as $H_{1}=\operatorname{diag}\{0.1,0.1,-0.05\}, F_{1}=I$ and $\Delta=\operatorname{diag}\left\{\delta_{1}, \delta_{2}, \delta_{3}\right\}$, where $\delta_{1}, \delta_{2}$ and $\delta_{3}$ vary randomly between -1 and 1 . Application of Theorem 2 gave the control law matrices shown in the second row of Table 1 . The simulation results for the controlled dynamics are shown in Fig. 2.

\subsection{Scenario 3: Time-varying fault and model with polytopic uncertainties}

In the motor model, $\phi_{m g}$ and $J$ are considered as uncertain parameters of the form

$$
\begin{aligned}
& \phi_{m g}=\xi_{1} \phi_{m g 1}+\xi_{2} \phi_{m g 2} \\
& J=\xi_{1} J_{1}+\xi_{2} J_{2}
\end{aligned}
$$

where $\left(\phi_{m g 1}, J_{1}\right)=(0.5,0.016),\left(\phi_{m g 2}, J_{2}\right)=(1.5,0.036)$, $\xi_{1}+\xi_{2}=1, \xi_{1}$ and $\xi_{2}$ are uncertain variables lying within $[0,1]$.

This gives a polytopic uncertainty with two vertices defined by the matrices:

Vertex 1:

$$
A_{1}=\left[\begin{array}{ccc}
0.9248 & 0.0591 & 0 \\
0 & 0 & 0 \\
0.9655 & 0.0595 & 1
\end{array}\right], B_{1}=\left[\begin{array}{c}
86.9355 \\
0 \\
87.5465
\end{array}\right] \text {. }
$$

Vertex 2:

$$
A_{2}=\left[\begin{array}{ccc}
0.9659 & 0.0822 & 0 \\
0 & 0 & 0 \\
0.9865 & 0.08 & 1
\end{array}\right], B_{2}=\left[\begin{array}{c}
120.8769 \\
0 \\
119.1818
\end{array}\right]
$$

The controlled system has been simulated with $\left(\phi_{m g}, J\right)=(0.5,0.016)$ and $\Gamma=0.7$ until $k=20$ when the parameters were switched to $\left(\phi_{m g}, J\right)=(1.5,0.036)$ and $\Gamma=(1+0.3 \sin (p))$, and finally at $k=40$, a further switch occurs to $\left(\phi_{m g}, J\right)=(0.5,0.016)$ and $\Gamma=(1+0.3 \cos (p))$. Application of Theorem 3 gave the control law matrices shown in the third row of Table 1 . The simulation results for the controlled dynamics are shown in Fig. 3.

From Figs. 1-3, it is seen that the tracking performance along the pass direction experiences degradation when the value of fault changes at $k=20$ and $k=40$. However, the tracking performance quickly recovers over subsequent passes.

Given the control law matrices $K_{1}$ and $K_{2}$, the control law for implementation on pass $k$ can, after routine manipulations of (9), be written as

$$
\begin{aligned}
u_{k}(p)= & u_{k-1}(p)+K_{1}\left(x_{k}(p)-x_{k-1}(p)\right)+ \\
& K_{3}\left(y_{r}(p+1)-y_{k-1}(p+1)\right)
\end{aligned}
$$

where the second term on the right-hand side is phase-lead ILC, i.e., information that is non-causal in the temporal domain. The first term on the right-hand side is activated by the difference in the state vectors on two successive passes and an obvious extension is to seek to replace the state vectors with their pass profile counterparts. Finally, there are alternative $2 \mathrm{D}$ systems based designs that could be employed, e.g., [28], but the form of the control law to be implemented is much less transparent in terms of actual implementation. Moreover, the analysis in [22] uses a repetitive process model that has no $2 \mathrm{D}$ discrete linear systems counterpart. Finally, the repetitive process setting for design can be undertaken for differential dynamics, i.e., where design by emulation is the preferred or only option for a given application. This uses the theory of differential repetitive processes and again there is no $2 \mathrm{D}$ systems model counterpart or lifted model. In comparison to [24], the major 


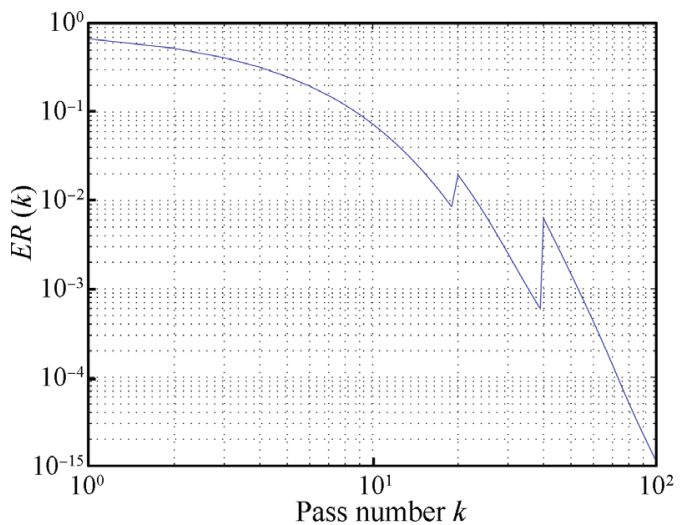

(a) Root mean square error

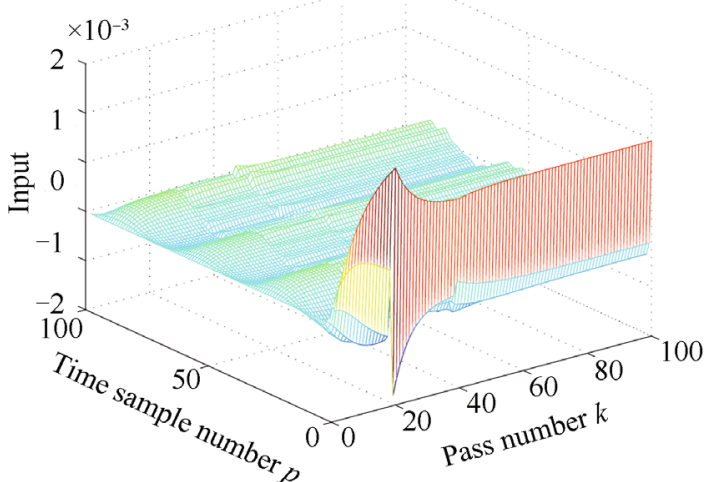

(c) Input progression

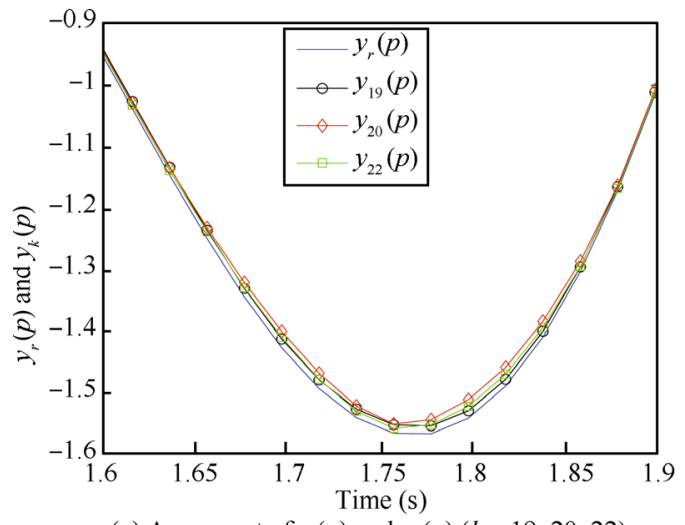

(e) A segment of $y_{r}(p)$ and $y_{K}(p)(k=19,20,22)$

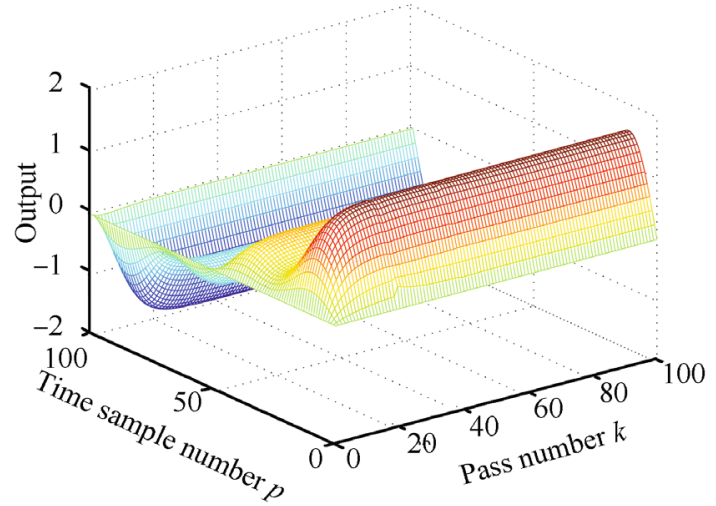

(b) Output progression

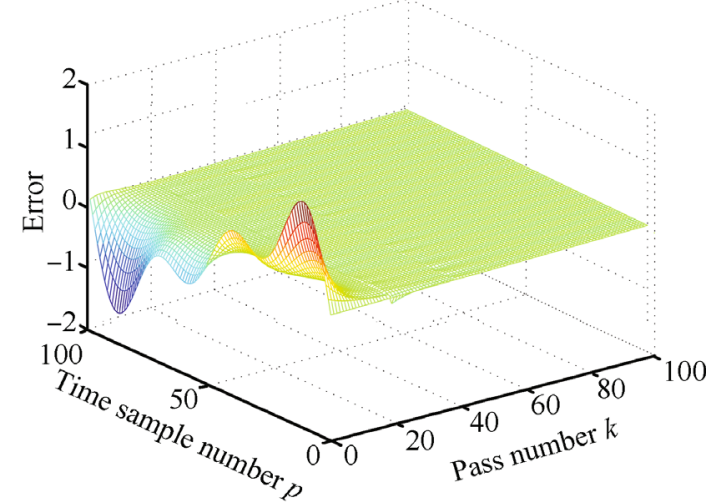

(d) Error progression

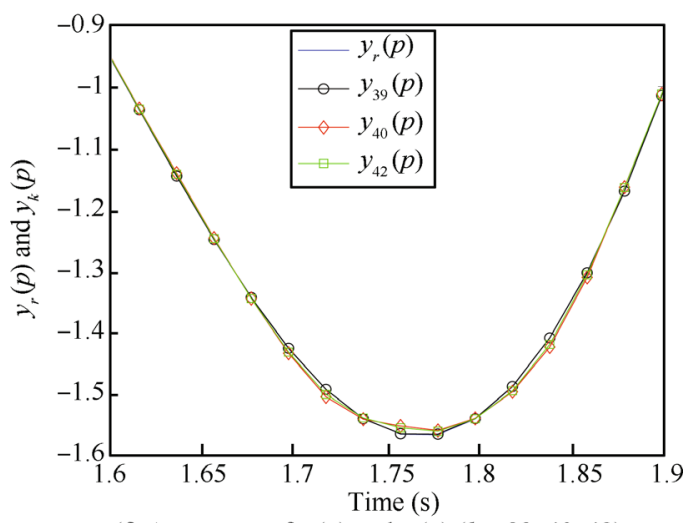

(f) A segment of $y_{r}(p)$ and $y_{k}(p)(k=39,40,42)$

Fig. 2 Simulation results for the Scenario 2 design

advance in this paper is the robustness analysis and control law design.

\section{Conclusions}

This paper has considered the relevant applications problem of ILC design in the presence of actuator faults. The new results have been derived by writing the dynamics in each case as a discrete linear repetitive process, resulting in LMI based computations. It has also been shown that the results extend in a natural manner to norm bounded and polytopic uncertainty. The performance has been illustrated by a simulation case study on an electric motor system used in other ILC designs. These results provide a firm basis for onward development and eventual experimental verification. 


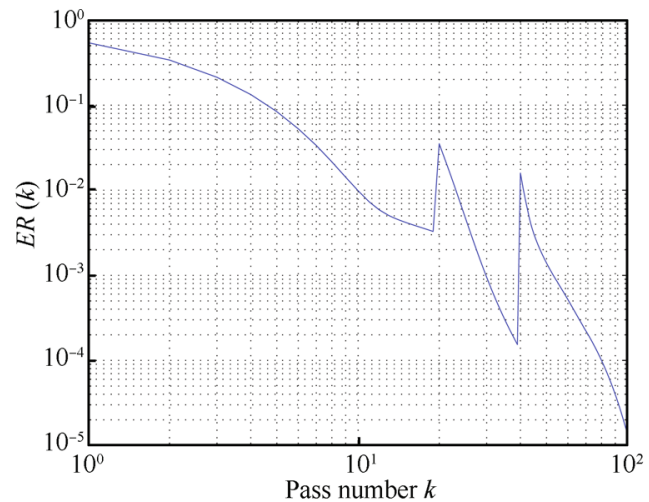

(a) Root mean square error

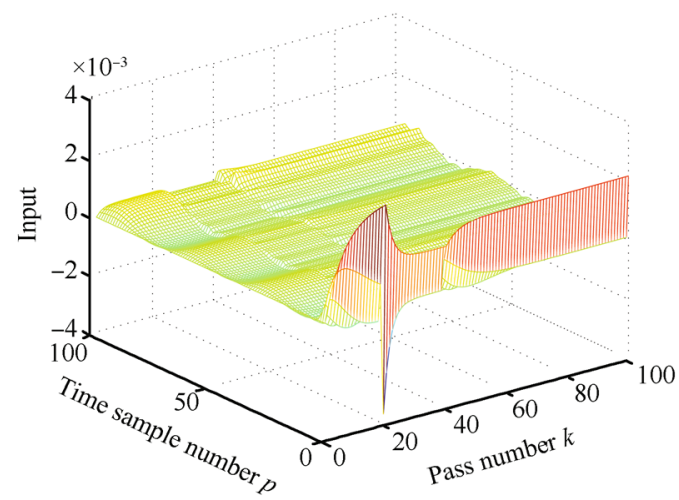

(c) Input progression

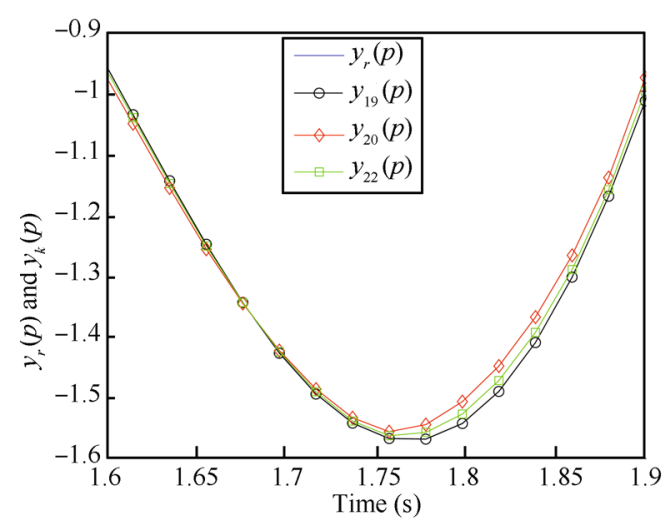

(e) A segment of $y_{r}(p)$ and $y_{K}(p)(k=19,20,22)$

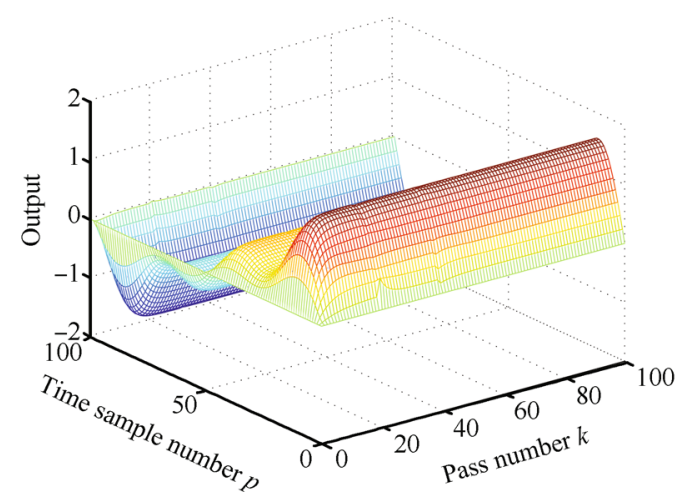

(b) Output progression

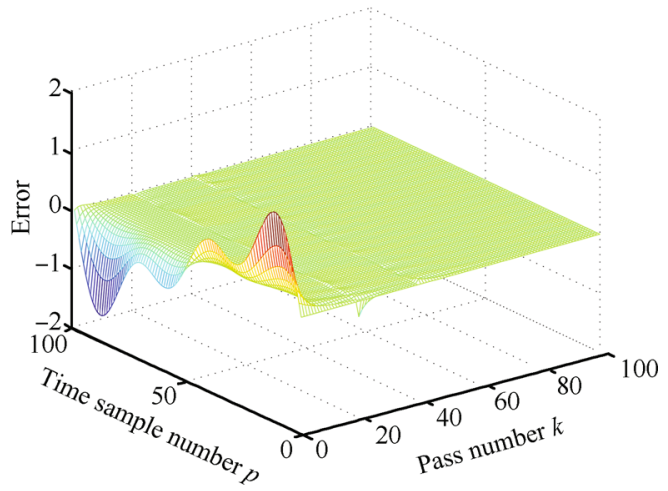

(d) Error progression

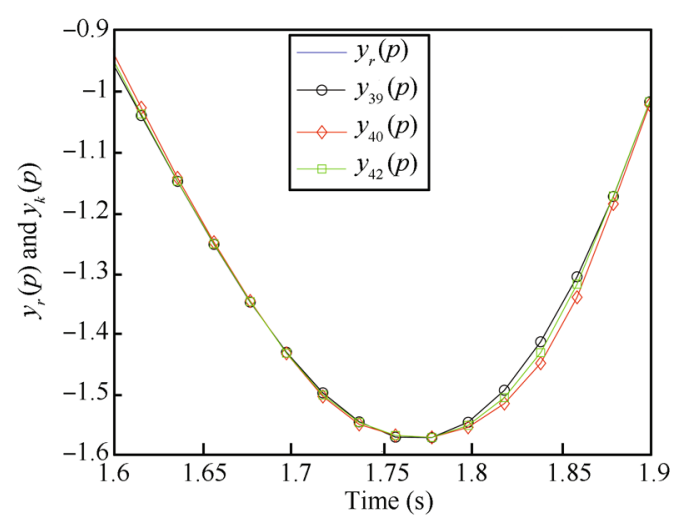

(f) A segment of $y_{r}(p)$ and $y_{k}(p)(k=39,40,42)$

Fig. 3 Simulation results for the Scenario 3 design

\section{References}

[1] S. Arimoto, S. Kawamura, F. Miyazaki. Bettering operation of robots by learning. Journal of Robotic Systems, vol.1, no. 2, pp. 123-140, 1984.

[2] H. S. Ahn, Y. Q. Chen, K. L. Moore. Iterative learning control: Brief survey and categorization. IEEE Transactions on Systems, Man, and Cybernetics, Part C: Applications and Reviews, vol. 37, no. 6, pp. 1099-1121, 2007.

[3] D. A. Bristow, M. Tharayil, A. G. Alleyne. A survey of iterative learning control. IEEE Control Systems Magazine, vol. 26, no. 3, pp. 96-114, 2006.

[4] D. Li, J. M. Li. Adaptive iterative learning control for nonlinearly parameterized systems with unknown time-varying delay and unknown control direction. International Journal of Automation and Computing, vol.9, no. 6, pp.578-586, 2012.

[5] Z. Q. Wu, C. H. Xu, Y. Yang. Robust iterative learning control of single-phase grid-connected inverter. International Journal of Automation and Computing, vol.11, no. 4, pp. 404-411, 2014.

[6] D. Y. Meng, Y. M. Jia, J. P. Du, F. S. Yu. Data-driven control for relative degree systems via iterative learning. IEEE Transactions On Neural Networks, vol. 22, no. 12, pp. 22132225, 2011.

[7] W. Chen, Y. Q. Chen, C. P. Yeh. Robust iterative learning control via continuous sliding-mode technique with valida- 
tion on an SRV02 rotary plant. Mechatronics, vol. 22, no. 5 pp. 588-593, 2012.

[8] X. D. Yu, Z. H. Xiong, D. X. Huang, Y. H. Jiang. Modelbased iterative learning control for batch processes using generalized hinging hyperplanes. Industrial \& Engineering Chemistry Research, vol. 52, no. 4, pp. 1627-1634, 2013.

[9] R. H. Chi, D. X. Zhang, X. M. Liu, Z. S. Hou, S. T. Jin. An anticipatory terminal iterative learning control approach with applications to constrained batch processes. Chinese Journal of Chemical Engineering, vol. 21, no. 3, pp. 271-275, 2013.

[10] H. Q. Sun, Z. S. Hou, D. Y. Li. Coordinated Iterative learning control schemes for train trajectory tracking with overspeed protection. IEEE Transactions on Automation Science and Engineering, vol. 10, no. 2, pp. 323-333, 2013.

[11] Z. S. Hou, J. W. Yan, J. X. Xu, Z. J. Li. Modified iterativelearning-control-based ramp metering strategies for freeway traffic control with iteration-dependent factors. IEEE Transactions on Intelligent Transportation Systems, vol. 13, no. 2, pp. 606-618, 2012.

[12] C. T. Freeman. Constrained point-to-point iterative learning control with experimental verification. Control Engineering Practice, vol. 20, no. 5, pp. 489-498, 2012.

[13] G. Parmar, K. Barten, S. Awtar. Large dynamic range nanopositioning using iterative learning control. Precision Engineering, vol. 38, no. 1, pp. 48-56, 2014.

[14] D. Q. Huang, J. X. Xu, V. Venkataramanan, T. C. T. Huynh. High-performance tracking of piezoelectric positioning stage using current-cycle iterative learning control with gain scheduling. IEEE Transactions on Industrial Electronics, vol. 61, no. 2, pp. 1085-1098, 2014.

[15] J. E. Kurek, M. B. Zaremba. Iterative learning control synthesis based on 2-D system theory. IEEE Transactions on Automatic Control, vol. 38, no. 1, pp. 121-125, 1993.

[16] J. Shi, F. R. Gao, T. J. Wu. Integrated design and structure analysis of robust iterative learning control system based on a two-dimensional model. Industrial \& Engineering Chemistry Research, vol. 44, no. 21, pp. 8095-8105, 2005.

[17] T. Liu, Y. Q. Wang. A synthetic approach for robust constrained iterative learning control of piecewise affine batch processes. Automatica, vol. 48, no. 11, pp. 2762-2775, 2012.

[18] E. Rogers, K. Gałkowski, D. H. Owens. Control systems theory and applications for linear repetitive processes. Lecture Notes in Control and Information Sciences 349, 2007.

[19] L. Hladowski, K. Gałkowski, Z. L. Cai, E. Rogers, C. T. Freeman, P. L. Lewin. Experimentally supported 2D systems based iterative learning control law design for error convergence and performance. Control Engineering Practice, vol. 18, no. 4, pp. 339-348, 2010.

[20] L. Hladowski, K. Gałkowski, Z. L. Cai, E. Rogers, C. T. Freeman, P. L. Lewin. Output information based iterative learning control law design with experimental verification. Journal of Dynamic Systems, Measurement, and Control, vol. 134, no. 2, Article number 021012, 2012.

[21] W. Paszke, E. Rogers, K. Gałkowski, Z. L. Cai. Robust finite frequency range iterative learning control design and experimental verification. Control Engineering Practice, vol. 21, no. 10, pp. 1310-1320, 2013.

[22] B. Cichy, K. Gałkowski, E. Rogers. 2D systems based robust iterative learning control using noncausal finite-time interval data. Systems \& Control Letters, vol.64, pp. 3642, 2014 .
[23] Y. Q. Wang, Y. Yang, D. H. Zhou, F. R. Gao. Active fault-tolerant control of nonlinear batch processes with sensor faults. Industrial \& Engineering Chemistry Research, vol. 46, no. 26, pp. 9158-9169, 2007.

[24] Y. Q. Wang, J. Shi, D. H. Zhou, F. R. Gao. Iterative learning fault-tolerant control for batch processes. Industrial \& Engineering Chemistry Research, vol. 45, no. 26, pp.90509060, 2006.

[25] L. M. Wang, S. Y. Mo, D. H. Zhou, F. R. Gao, X. Chen. Robust delay dependent iterative learning fault-tolerant control for batch processes with state delay and actuator failures. Journal of Process Control, vol.22, no. 7, pp. 12731286, 2012.

[26] Y. Q. Wang, D. H. Zhou, F. R. Gao. Iterative learning reliable control of batch processes with sensor faults. Chemical Engineering Science, vol.63, no. 4, pp. 1039-1051, 2008.

[27] L. H. Xie. Output feedback $H_{\infty}$ control of systems with parameter uncertainty. International Journal of Control, vol. 63, no. 4, pp. 741-750, 1996.

[28] J. Shi, F. R. Gao, T. J. Wu. Robust design of integrated feedback and iterative learning control of a batch process based on a 2D Roesser system. Journal of Process Control, vol. 15, no. 8, pp. 907-924, 2005.

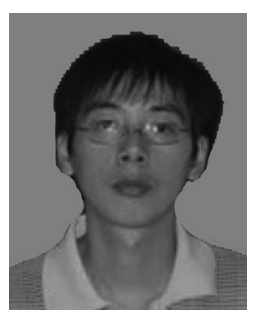

Jian Ding received the M.Sc. degree from College of Internet of Things, Jiangnan University, China in 2007. He is now a $\mathrm{Ph}$. D. candidate at the Key Laboratory of Advanced Process Control for Light Industry (Ministry of Education), Jiangnan University, China.

His research interest is iterative learning control for dynamical system with actuator

and sensor failures.

E-mail: 443234365@qq.com

ORCID iD: 0000-0003-0348-5894

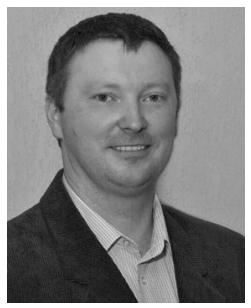

Blazej Cichy received the M. Sc. degree from University of Zielona Gora, Poland in 2003, and the Ph. D. degree from Wroclaw University of Technology, Poland in 2011. Currently, he is an associate professor at the University of Zielona Gora.

His research interests include theory and applications of control systems and especially in application of iterative learning control.

E-mail: B.Cichy@issi.uz.zgora.pl

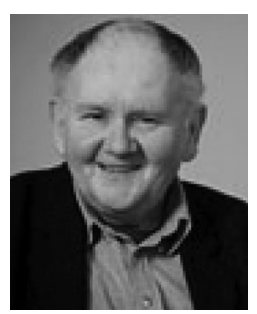

Krzysztof Galkowski is a professor of technical sciences in University of Zielona Gora, Institute of Control and Computation Engineering and also in Nicolaus Copernicus University, Institute of Physics, Poland. He is a member of the editorial board of Multidimensional Systems and Signal Processing (Springer) and International Journal of Control and a associate editor of IET Control Theory and Applications. He is the coauthor/editor of 4 monographs/books, a few special issues of leading international journals, e.g., International Journal of Control, Multidimensional Systems and Signal Processing, Asian Journal of Control, and over 100 papers in leading peer reviewed journals and about 200 in the proceedings of international conferences. He has co-organised a series of international workshops on multidimensional systems. He is a visiting professor in the School 
of Electronics and Computer Science, University of Southampton, UK, was three times a Gerhard Mercator guest professor, funded by Deutsche Forschungsgemeinschaft (DFG), in the University of Wuppertal, Germany (Nos. 09.2004-08.2005, 10.200609.2007 and 03.2011-02.2012). From 2011 to 2014, he has been a chair professor in East China University of Science and Technology, China.

His research interests include multidimensional systems and repetitive processes - theory and applications, control, iterative learning control and related numerical and symbolic algebra methods.

E-mail: K.Galkowski@issi.uz.zgora.pl

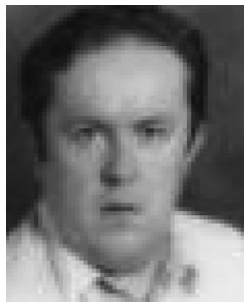

Eric Rogers received the B.Sc. degree in mechanical engineering from Queen's University Belfast, UK, received the M. Sc. and $\mathrm{Ph}$. D. degrees in control systems from the University of Sheffield, UK, and received the Doctor of Science degree in control systems from Queens University Belfast, UK. He is a professor of control systems theory and design in School of Electronics and Computer Science, the University of Southampton
UK. He is the editor-in-chief of the International Journal of Control and has an extensive record of service on the international programme and organizing committees of international control conferences and acts as a consultant to government grant awarding agencies and industries in the UK and elsewhere.

His research interests include iterative learning, repetitive control theory and experimental benchmarking, robotic-assisted stroke rehabilitation, multidimensional systems theory, flow control and autonomous underwater vehicles control.

E-mail: etar@ecs.soton.ac.uk

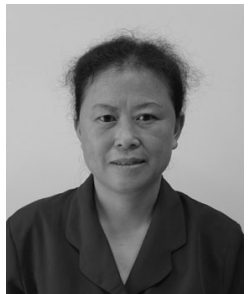

Hui-Zhong Yang received the $\mathrm{Ph}$. D degree in control theory and control engineering from East China University of Science and Technology, China in 2001. She is now a professor at the Key Laboratory of Advanced Process Control for Light Industry (Ministry of Education), Jiangnan University, China.

Her research interests include modeling and control of complex industrial process.

E-mail: yhz@jiangnan.edu.cn (Corresponding author)

ORCID iD: 0000-0001-8511-9251 